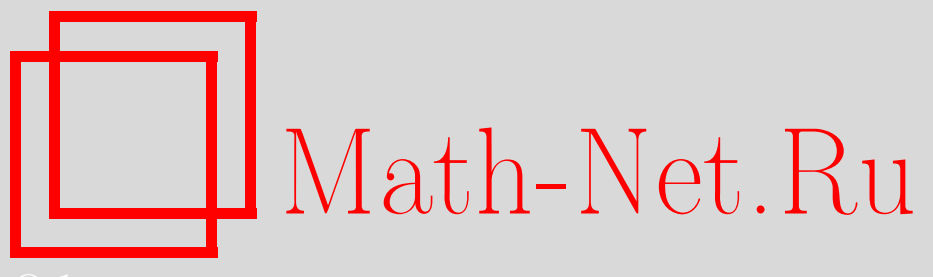

А. С. Холево, Классические пропускные способности квантового канала с ограничением на входе, Теория вероятн. и ее примен., 2003, том 48, выпуск 2, 359374

DOI: https://doi.org/10.4213/tvp289

Использование Общероссийского математического портала Math-Net.Ru подразумевает, что вы прочитали и согласны с пользовательским соглашением

http://www . mathnet.ru/rus/agreement

Параметры загрузки:

IP : 3.85 .183 .62

26 апреля 2023 г., 15:45:40

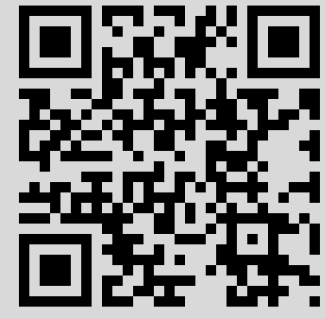




\title{
КЛАССИЧЕСКИЕ ПРОПУСКНЫЕ СПОСОБНОСТИ КВАНТОВОГО КАНАЛА С ОГРАНИЧЕНИЕМ НА ВХОДЕ
}

\begin{abstract}
В этой статье мы восполняем пробел в предыдущих работах и даем доказательство формулы для пропускной способности с использованием сцепленного состояния (entanglement-assisted capacity) для квантового канала с ограничением на входе (такого как бозонный гауссовский канал). Доказательство основано на теореме кодирования для классического-квантового канала с ограничением на входе и на конечномерной аппроксимации входных операторов плотности. Даются также достаточные условия достижимости супремумов в формулах для пропускных способностей.
\end{abstract}

Ключевые слова и фразы: канал связи квантовый, классическая пропускная способность, сцепленное состояние.

Выражение для пропускной способности с использованием сцепленного состояния для квантового канала с шумом, представляющее ее как максимум взаимной информации по всевозможным входным состояниям, было получено в [1], [2] в случае каналов в конечномерном гильбертовом пространстве. Альтернативное доказательство было дано в [3], см. также [4]. В [2], [5] подходящим образом модифицированная формула была применена к квантовым гауссовским каналам, которые являются наиболее важным примером каналов с ограничением на входе, действующих в бесконечномерном гильбертовом пространстве. В этой статье мы восполняем пробел в предыдущих работах и даем доказательство гипотетической формулы (34). Доказательство основано на теореме кодирования для классического-квантового канала с ограничением на входе и на конечномерной аппроксимации входных операторов плотности. Даются также достаточные условия достижимости супремумов в формулах для пропускных способностей. Объяснение используемых далее понятий квантовой теории информации см. в [4].

1. Сначала рассмотрим случай классического-квантового (c-q) канала с дискретным (возможно, бесконечным) алфавитом $\mathscr{X}$. Для любого $x \in \mathscr{X}$ задан оператор плотности $S_{x}$ в гильбертовом пространстве $\mathscr{H}$

* Математический институт им. В. А. Стеклова РАН, ул. Губкина, 8, 119991 Москва, Россия; e-mail: holevo@mi.ras.ru

1) Работа выполнена при поддержке ИНТАС (грант № 00-738). 
(вообще говоря, бесконечномерном) с конечной энтропией фон Неймана $H\left(S_{x}\right)$ (см. Приложение). C-q канал задается отображением $x \rightarrow S_{x}$. Пусть $f(x)$ - неотрицательная непостоянная функция на входном алфавите. Переходя к блочному кодированию, введем аддитивное ограничение на входные слова $w=\left(x_{1}, \ldots, x_{n}\right)$, потребовав, чтобы

$$
f\left(x_{1}\right)+\cdots+f\left(x_{n}\right) \leqslant n E,
$$

где $E$ - положительная постоянная. Классическая пропускная способность такого канала была определена и вычислена в [6] при условии равномерной ограниченности энтропий $H\left(S_{x}\right)$. В настоящей работе это условие неудобно; используя те же рассуждения, можно доказать

Предложение 1. Обозначим ЯР класс конечных входных распределений $\pi=\left\{\pi_{x}\right\}$, удовлетворяюших условию

$$
\sum_{x} \pi_{x} f(x) \leqslant E,
$$

и предположим, что $\mathscr{P}$ непуст и канал удовлетворяет условию:

$$
\sup _{\pi \in \mathscr{P}} H\left(\sum_{x} \pi_{x} S_{x}\right)<\infty .
$$

Тогда классическая пропускная способность канала $x \rightarrow S_{x}$ с ограничением (1) конечна и дается выражением

$$
C=\sup _{\pi \in \mathscr{P}}\left[H\left(\sum_{x} \pi_{x} S_{x}\right)-\sum_{x} \pi_{x} H\left(S_{x}\right)\right] .
$$

Величина в квадратных скобках часто возникает в вопросах, связанных с пропускной способностью, и обычно обозначается

$$
\chi\left(\left\{\pi_{x}\right\},\left\{S_{x}\right\}\right)=H\left(\sum_{x} \pi_{x} S_{x}\right)-\sum_{x} \pi_{x} H\left(S_{x}\right) .
$$

Используя квантовую относительную энтропию $H(\cdot ; \cdot)$ (см. Приложение), можно записать эту величину в виде выражения

$$
\chi\left(\left\{\pi_{x}\right\},\left\{S_{x}\right\}\right)=\sum_{x} \pi_{x} H\left(S_{x} ; \sum_{y} \pi_{y} S_{y}\right),
$$

определенного однозначно и принимающего значения в диапазоне $[0,+\infty]$, даже если энтропии, входящие в формулу (5), бесконечны. Предложение 1 может быть обобщено на этот случай, см. [13].

Пусть теперь входной алфавит $\mathscr{X}$ является сепарабельным локально компактным пространством. Рассмотрим канал, задаваемый 
слабо непрерьвным отображением $x \rightarrow S_{x}, x \in \mathscr{X}$ (слабая непрерывность означает непрерывность всех матричных элементов $\left\langle\psi\left|S_{x}\right| \phi\right\rangle$, $\psi, \phi \in \mathscr{H}$ ). Отметим, что слабая сходимость в множестве операторов плотности эквивалентна сходимости по следовой норме [7]. Для произвольной борелевской меры $\pi$ на $\mathscr{X}$ обозначим

$$
\bar{S}_{\pi}=\int_{\mathscr{X}} S_{x} \pi(d x)
$$

В силу непрерывности функции $S_{x}$ интеграл определен и задает оператор плотности в $\mathscr{H}$. В предположении $H\left(\bar{S}_{\pi}\right)<\infty$ имеем

$$
\chi(\pi) \equiv H\left(\bar{S}_{\pi}\right)-\int_{\mathscr{X}} H\left(S_{x}\right) \pi(d x)=\int_{\mathscr{X}} H\left(S_{x} ; \bar{S}_{\pi}\right) \pi(d x) \geqslant 0,
$$

где функции $H\left(S_{x}\right), H\left(S_{x} ; \bar{S}_{\pi}\right)$ неотрицательны и полунепрерывны снизу [8] и потому интегралы определены. Мы предположим, что $f-$ борелевская функция, и рассмотрим подмножество $\mathscr{P}^{B}$ борелевских вероятностных мер $\pi$ на $\mathscr{X}$, удовлетворяющих условию

$$
\int_{\mathscr{X}} f(x) \pi(d x) \leqslant E .
$$

Предложение 2. Пусть функчия $f$ полунепрерьвна снизу и стремится $\kappa+\infty$ на бесконечности, и пусть существует самосопряженный оператор $F$, удовлетворяющий условию

$$
\operatorname{Tr} \exp (-\beta F)<\infty \quad \text { для всех } \beta>0,
$$

такой, что

$$
f(x) \geqslant \operatorname{Tr} S_{x} F, \quad x \in \mathscr{X} .
$$

Тогда пропускная способность $C$ конечна и

$$
C=\max _{\pi \in \mathscr{P}_{B}} \chi(\pi) .
$$

Д о к а з а т е л ь с т в о. Из условия (9) вытекает, что оператор $F$ полуограничен снизу; для простоты предположим, что $F \geqslant 0$, однако общий случай сводится к этому. Тогда правая часть (10) определяется как в соо'тношении (14) ниже. Обозначая

$$
S_{\beta}=[\operatorname{Tr} \exp (-\beta F)]^{-1} \exp (-\beta F),
$$

имеем

$$
\beta \operatorname{Tr} \bar{S}_{\pi} F-H\left(\bar{S}_{\pi}\right)=H\left(\bar{S}_{\pi} ; S_{\beta}\right)-\ln \operatorname{Tr} \exp (-\beta F)
$$

откуда, используя (10), получаем

$$
H\left(\bar{S}_{\pi}\right) \leqslant \beta \operatorname{Tr} \bar{S}_{\pi} F+\ln \operatorname{Tr} \exp (-\beta F) \leqslant \beta E+\ln \operatorname{Tr} \exp (-\beta F) .
$$


Таким образом, условие (3) выполнено, $C$ конечна и дается соотношением (4). В предположениях, что отображение $x \rightarrow S_{x}$ слабо непрерывно, функция $f$ полунепрерывна снизу и выполняется условие (3), предложение 2 из работы [6] влечет

$$
C=\sup _{\pi \in \mathscr{P}_{B}} \chi(\pi)
$$

и мы хотим доказать, что супремум достигается.

В множестве всех борелевских вероятностных мер на $\mathscr{X}$ введем топологию слабой сходимости: последовательность $\pi^{(l)}(d x)$ сходится слабо к $\pi(d x)$, если

$$
\int_{\mathscr{X}} g(x) \pi^{(l)}(d x) \rightarrow \int_{\mathscr{X}} g(x) \pi(d x)
$$

для всех ограниченных непрерывных функций $g$ на $\mathscr{X}$. Тогда множество $\mathscr{P}^{B}$ замкнуто, поскольку для полунепрерывной снизу функции $f$

$$
\lim _{l \rightarrow \infty} \int_{\mathscr{X}} f(x) \pi^{(l)}(d x) \geqslant \int_{\mathscr{X}} f(x) \pi(d x) .
$$

Более того, оно равномерно плотно: для любого компакта $\mathscr{K} \subset \mathscr{X}$ имеем $\pi(\mathscr{X} \backslash \mathscr{K}) \leqslant E / \inf _{x \in \mathscr{X} \backslash \mathscr{K}} f(x)$ для $\pi \in \mathscr{P}^{B}$. Используя общий критерий относительной слабой компактности [9], получаем, что $\mathscr{P}^{B}$ слабо компактно.

Докажем, что функция $\chi(\pi)$ полунепрерывна сверху и, значит, достигает максимума на $\mathscr{P}^{B}$. Отображение $\pi \rightarrow \bar{S}_{\pi}$ непрерывно в слабой операторной топологии и, следовательно, в топологии следовой нормы. Рассмотрим первое слагаемое в формуле (7). Поскольку квантовая энтропия полунепрерывна снизу, функция $\pi \rightarrow H\left(\bar{S}_{\pi}\right)$ также полунепрерывна снизу. Покажем, что она полунепрерывна сверху и, значит, непрерывна на множестве $\mathscr{P}^{B}$. Согласно $(12),(10)$, имеем

$$
H\left(\bar{S}_{\pi}\right) \geqslant \limsup _{n \rightarrow \infty} H\left(\bar{S}_{\pi^{n}}\right)-\beta \limsup \operatorname{Tr} \bar{S}_{\pi^{n}} F \geqslant \limsup _{n \rightarrow \infty} H\left(\bar{S}_{\pi^{n}}\right)-\beta E
$$

для произвольной последовательности $\left\{\pi^{n}\right\} \in \mathscr{P}^{B}$, слабо сходящейся к $\pi$. Полагая $\beta \rightarrow 0$, получаем полунепрерывность сверху.

Второе слагаемое в (7) полунепрерывно сверху как точная нижняя грань семейства непрерывных функций $\pi \rightarrow-\int g(x) \pi(d x)$, где $g$ пробегает ограниченные непрерывные функции, удовлетворяющие условию $0 \leqslant g(x) \leqslant H\left(S_{x}\right), x \in \mathscr{X}$. Поэтому (7) полунепрерывно сверху, и утверждение доказано.

2. Теперь рассмотрим (квантовый-квантовый или кратко q-q) канал $\Phi$ в гильбертовом пространстве $\mathscr{H}$, т.е. сохраняющее след линейное вполне положительное отображение операторов в $\mathscr{H}$. Дадим определение пропускной способности при аддитивном ограничении на входе 
этого канала. Пусть $F$ - самосопряженный положительный, вообще говоря, неограниченный оператор в $\mathscr{H}$, не пропорциональный единичному, представляющий наблюдаемую, среднее значение которой подлежит ограничению (например, энергию системы). Для произвольного оператора плотности $S$ со спектральным разложением $S=\sum_{j=1}^{\infty} \lambda_{j}\left|e_{j}\right\rangle\left\langle e_{j}\right|$ положим

$$
\operatorname{Tr} S F:=\sum_{j=1}^{\infty} \lambda_{j}\left\|\sqrt{F} e_{j}\right\|^{2} \leqslant+\infty,
$$

считая, что $\left\|\sqrt{F} e_{j}\right\|^{2}=+\infty$, если $e_{j}$ не принадлежит области определения $\sqrt{F}$. Используя спектральное разложение $F=\int_{0}^{\infty} x d E(x)$, где $E(x)$ - спектральная функция оператора $F$, мы можем записать это выражение в другой форме:

$$
\operatorname{Tr} S F=\int_{0}^{\infty} x d \operatorname{Tr} S E(x) .
$$

Введем аналог условия (3):

$$
\sup _{S: \operatorname{Tr} S F \leqslant E} H(\Phi[S])<\infty
$$

где $E-$ положительная постоянная.

Для канала $\Phi^{\otimes n}$ в $\mathscr{H}^{\otimes n}$ соответствующая наблюдаемая есть

$$
F^{(n)}=F \otimes \cdots \otimes I+\cdots+I \otimes \cdots \otimes F
$$

Потребуем, чтобы входные состояния $S^{(n)}$ канала $\Phi^{\otimes n}$ удовлетворяли аддитивному ограничению

$$
\operatorname{Tr} S^{(n)} F^{(n)} \leqslant n E .
$$

Заметим, что условие (16) влечет аналогичное свойство канала $\Phi^{\otimes n}$ :

$$
\sup _{S^{(n)}: \operatorname{Tr} S^{(n)} F^{(n)} \leqslant n E} H\left(\Phi^{\otimes n}\left[S^{(n)}\right]\right)<\infty .
$$

В самом деле, из субаддитивности квантовой энтропии по отношению к тензорным произведениям следует

$$
H\left(\Phi^{\otimes n}\left[S^{(n)}\right]\right) \leqslant \sum_{k=1}^{n} H\left(\Phi\left[S_{k}^{(n)}\right]\right),
$$

где $S_{k}^{(n)}$ есть $k$-е частичное состояние $S^{(n)}$. Также в силу вогнутости энтропии

$$
\sum_{k=1}^{n} H\left(\Phi\left[S_{k}^{(n)}\right]\right) \leqslant n H\left(\Phi\left[\bar{S}^{(n)}\right]\right)
$$


где $\bar{S}^{(n)}=n^{-1} \sum_{k=1}^{n} S_{k}^{(n)}$. Используя (15), неравенство (17) можно переписать в виде

$$
\frac{1}{n} \sum_{k=1}^{n} \operatorname{Tr} S_{k}^{(n)} F=\operatorname{Tr} \bar{S}^{(n)} F \leqslant E,
$$

откуда следует, что

$$
\sup _{S^{(n)}: \operatorname{Tr} S^{(n)} F^{(n)} \leqslant n E} H\left(\Phi^{\otimes n}\left[S^{(n)}\right]\right) \leqslant n \sup _{S: \operatorname{Tr} S F \leqslant E} H(\Phi[S]) .
$$

О п р е д е л е н и е. Мы называем кодом $\left(\Sigma^{(n)}, M^{(n)}\right)$ длины $n$ и размера $N$ семейство $\Sigma^{(n)}=\left\{S_{w}^{(n)} ; w=1, \ldots, N\right\}$ состояний, удовлетворяющих ограничению (17), вместе с разложением единицы $M^{(n)}=$ $\left\{M_{j}^{(n)} ; j=0,1, \ldots, N\right\}$ в $\mathscr{H}^{\otimes n}$. Вероятность ошибки такого кода равна

$$
P_{e}\left(\Sigma^{(n)}, M^{(n)}\right)=\max _{w=1, \ldots, N}\left\{1-\operatorname{Tr} \Phi^{\otimes n}\left[S_{w}^{(n)}\right] M_{w}^{(n)}\right\}
$$

а минимальная вероятность ошибки по всевозможным кодам длины $n$ и размера $N$ обозначается $p_{e}(n, N)$. Классическая пропускная способность $C(\Phi)$ есть точная верхняя грань скоростей передачи $R$, для котоpыx $\lim _{n \rightarrow \infty} p_{e}\left(n, e^{n R}\right)=0$.

Обозначим через $\mathfrak{S}^{(n)}$ множество состояний в $\mathscr{H}^{\otimes n}$, удовлетворяющих условию (17), а через $\mathscr{P}^{(n)}$ множество пар $\left(\pi^{(n)}, \Sigma^{(n)}\right)$, где $\pi_{w}^{(n)}$ вероятности состояний $S_{w}^{(n)}$, удовлетворяющих условию

$$
\sum_{w=1}^{N} \pi_{w}^{(n)} \operatorname{Tr} S_{w}^{(n)} F^{(n)} \leqslant n E .
$$

Если задано распределение вероятностей $\pi^{(n)}=\left\{\pi_{w}^{(n)}\right\}$ на входных состояниях $S_{w}^{(n)}$, то, используя переходную вероятность $p(j \mid w)=$ $\operatorname{Tr} \Phi^{\otimes n}\left[S_{w}^{(n)}\right] M_{j}^{(n)}$ мы можем найти совместное распределение входа и выхода, вычислить шенноновскую информацию $\mathscr{I}_{n}\left(\pi^{(n)}, \Sigma^{(n)}, M^{(n)}\right)$ и определить величину

$$
\bar{C}^{(n)}(\Phi)=\sup _{\left(\pi^{(n)}, \Sigma^{(n)}\right) \in \mathscr{P}(n)} \chi\left(\left\{\pi_{w}^{(n)}\right\},\left\{\Phi^{\otimes n}\left[S_{w}^{(n)}\right]\right\}\right) .
$$

Если $\Sigma^{(n)} \subset \mathfrak{S}^{(n)}$, то $\left(\pi^{(n)}, \Sigma^{(n)}\right) \in \mathscr{P}^{(n)}$, и

$$
\mathscr{I}_{n}\left(\pi^{(n)}, \Sigma^{(n)}, M^{(n)}\right) \leqslant \bar{C}^{(n)}(\Phi)
$$

в силу квантовой энтропийной границы [6].

Предложение 3. Пусть канал $\Phi$ удовлетворяет условию (16). Тогда классическая пропускная способность этого канала при ограничении (17) конечна и равна

$$
\begin{aligned}
C(\Phi) & =\lim _{n \rightarrow \infty} \frac{1}{n} \sup _{\pi^{(n)}, \Sigma^{(n)} \subset \mathfrak{S}^{(n)}, M^{(n)}} \mathscr{I}_{n}\left(\pi^{(n)}, \Sigma^{(n)}, M^{(n)}\right) \\
& =\lim _{n \rightarrow \infty} \frac{1}{n} \bar{C}^{(n)}(\Phi) .
\end{aligned}
$$


Д о к а з а т е л ь с т в о. Соотношение (21) вытекает из классической теоремы кодирования. Неравенство $\leqslant$ в $(22)$ вытекает тогда из $(20)$. Покажем, что

$$
C(\Phi) \geqslant \lim _{n \rightarrow \infty} \frac{1}{n} \bar{C}^{(n)}(\Phi) \equiv \bar{C}(\Phi) .
$$

Пусть $R<\bar{C}(\Phi)$, тогда можно выбрать $n_{0}$, распределение вероятностей $\pi^{\left(n_{0}\right)}=\left\{\pi_{w}^{\left(n_{0}\right)}\right\}$ и семейство состояний $\Sigma^{\left(n_{0}\right)}=\left\{S_{w}^{\left(n_{0}\right)}\right\}$ в $\mathscr{H}^{\otimes n_{0}}$ такие, что $\left(\pi^{\left(n_{0}\right)}, \Sigma^{\left(n_{0}\right)}\right) \in \mathscr{P}^{\left(n_{0}\right)}$ и

$$
n_{0} R<\chi\left(\left\{\pi_{w}^{\left(n_{0}\right)}\right\},\left\{\Phi^{\otimes n_{0}}\left[S_{w}^{\left(n_{0}\right)}\right]\right\}\right)
$$

Рассмотрим с-q канал $\widetilde{\Phi}$ в $\mathscr{H}^{\otimes n_{0}}$, задаваемый формулой

$$
\tilde{\Phi}[S]=\sum_{w} \Phi^{\otimes n_{0}}\left[S_{w}\right]\left\langle e_{w} \mid S e_{w}\right\rangle
$$

и определим функцию ограничения для этого канала как $f(w)=$ $\operatorname{Tr} S_{w}^{\left(n_{0}\right)} F^{\left(n_{0}\right)}$. Условие (18) влечет $\sup _{\pi} H\left(\sum_{w} \pi_{w} \Phi^{\otimes n_{0}}\left[S_{w}\right]\right)<\infty$, где верхняя грань берется по множеству распределений вероятностей $\pi$, удовлетворяющих неравенству

$$
\sum_{w} \pi_{w} f(w) \leqslant n_{0} E
$$

т.е. условию (3). Согласно предложению 1 , пропускная способность канала $\widetilde{\Phi}$ равна

$$
C(\widetilde{\Phi})=\sup _{\pi} \chi\left(\left\{\pi_{w}\right\},\left\{\Phi^{\otimes n_{0}}\left[S_{w}^{\left(n_{0}\right)}\right]\right\}\right),
$$

где состояния фиксированы и верхняя грань берется по множеству распределений вероятностей $\pi$, удовлетворяющих (25). Согласно (24), это больше, чем $n_{0} R$. Обозначая $\widetilde{p}_{e}(n, N)$ минимальную вероятность ошибки для канала $\widetilde{\Phi}$, имеем

$$
p_{e}\left(n n_{0}, e^{\left(n n_{0}\right) R}\right) \leqslant \widetilde{p}_{e}\left(n, e^{n\left(n_{0} R\right)}\right),
$$

поскольку всякий код размера $N$ для канала $\widetilde{\Phi}$ является также кодом того же размера для $\Phi$. В самом деле, если $\widetilde{w}=\left(w_{1}, \ldots, w_{n}\right)$ - кодовое слово для канала $\widetilde{\Phi}$, то оно удовлетворяет ограничению $f\left(w_{1}\right)+\ldots+f\left(w_{n}\right) \leqslant$ $n n_{0} E$. Вводя состояние $S_{\widetilde{w}}^{\left(n n_{0}\right)}=S_{w_{1}}^{\left(n_{0}\right)} \otimes \ldots \otimes S_{w_{n}}^{\left(n_{0}\right)}$, мы видим, что это эквивалентно неравенству $\operatorname{Tr} S_{\widetilde{w}}^{\left(n n_{0}\right)} F^{\left(n n_{0}\right)} \leqslant n n_{0} E$, т.е., ограничению (17) для q-q канала $\Phi^{\otimes n n_{0}}$. Таким образом, выбрав $R<\bar{C}(\Phi)$, мы получаем, что правая, а следовательно, и левая части неравенства (26) стремятся к нулю при $n \rightarrow \infty$. Для произвольного $n^{\prime}$ можно найти $n$ такое, что $n n_{0} \leqslant n^{\prime} \leqslant(n+1) n_{0}$. Тогда

$$
p_{e}\left(n^{\prime}, e^{n^{\prime} R}\right) \leqslant p_{e}\left(n n_{0}, e^{(n+1) n_{0} R}\right) \leqslant p_{e}\left(n n_{0}, e^{\left(n n_{0}\right) R^{\prime}}\right) \longrightarrow 0,
$$


если $R^{\prime}$ выбрано так, что $R(1+1 / n) \leqslant R^{\prime}<\bar{C}(\Phi)$ для достаточно больших $n$. Это доказывает соотношение (23). Предложение 3 доказано.

Эти результаты поднимают вопросы, на которые пока нет ответа. Неизвестно, выполняется ли свойство аддитивности $\bar{C}^{(n)}(\Phi)=n \bar{C}^{(1)}(\Phi)$, из которого вытекало бы $C(\Phi)=\bar{C}^{(1)}(\Phi)$. Эта проблема является не менее трудной, чем до сих пор не решенная аналогичная проблема для каналов без ограничений (см. комментарии в [11]). Величина

$$
\bar{C}^{(1)}(\Phi)=\sup _{\sum_{i} \pi_{i} \operatorname{Tr} S_{i} F \leqslant E} \chi\left(\left\{\pi_{i}\right\},\left\{\Phi\left[S_{i}\right]\right\}\right)
$$

представляется доступной для вычисления, хотя даже для простейшего квантового гауссовского канала есть лишь естественное предположение о ее значении и о решении соответствующей проблемы максимизации (см. более подробно в [5]). Этот канал описывает «квантовый сигнал на фоне классического шума» и задается в картине Гейзенберга преобразованием

$$
a \rightarrow a+\xi
$$

где $a$ - оператор уничтожения данной моды, а $\xi$ - классическая комплексная гауссовская случайная величина с нулевым средним и дисперсией $N$ (среднее число шумовых фотонов). Ограничение на входе имеет вид $\operatorname{Tr} S a^{\dagger} a \leqslant E$, где $S$ - оператор плотности сигнала̇ $a$. Имеется гипотеза, что верхняя грань в (27) реализуется непрерывным комплексным гауссовским распределением

$$
\pi\left(d^{2} z\right)=(\pi E)^{-1} \exp \left(-|z|^{2} E^{-1}\right) d^{2} z, \quad z \in \mathbf{C}
$$

на когерентных состояниях $S_{z}=|z\rangle\langle z|$ и имеет величину

$$
\bar{C}^{(1)}(\Phi)=g(N+E)-g(N),
$$

где $g(x)=(x+1) \ln (x+1)-x \ln x$.

3. Теперь обратимся к пропускной способности с использованием сцепленного состояния. Рассмотрим следуюший протокол передачи классической информации через канал $\Phi$. Системы $A$ и $B$ описываются (чистым) сцепленным состоянием $S_{A B}$. Мы предполагаем, что количество сцепленности не ограничено, но конечно, т.е. $H\left(S_{A}\right)=H\left(S_{B}\right)<\infty$. Система $A$ производит кодирование $i \rightarrow \mathscr{E}_{i}$, зависящее от классического сигнала $i$, с вероятностями $\pi_{i}$ и посылает через канал системе $B$ свою часть общего состояния. Обозначим $\operatorname{Id}_{B}$ идеальный канал, т.е. тождественное отображение, для системы $B$. Таким образом, $B$ получает состояния $\left(\Phi \otimes \operatorname{Id}_{B}\right)\left[S_{i}\right]$, где $S_{i}=\left(\mathscr{E}_{i} \otimes \operatorname{Id}_{B}\right)\left[S_{A B}\right]$, с вероятностями $\pi_{i}$ и старается извлечь максимальную информацию, производя измерение над полученным состоянием. Применение блочного кодирования означает, 
что все это описание относится к каналу $\Phi^{\otimes n}$. Тогда сигнальные состояния $S_{w}^{(n)}$, передаваемые через канал $\Phi^{\otimes n} \otimes \operatorname{Id}_{B}^{\otimes n}$, имеют специальный вид

$$
S_{w}^{(n)}=\left(\mathscr{E}_{w}^{(n)} \otimes \operatorname{Id}_{B}^{\otimes n}\right)\left[S_{A B}^{(n)}\right]
$$

где $S_{A B}^{(n)}$ - чистое сцепленное состояние для $n$ экземпляров системы $A B$, удовлетворяющее условию $H\left(S_{B}^{(n)}\right)<\infty$, и $w \rightarrow \mathscr{E}_{w}^{(n)}-$ кодирования для $n$ экземпляров системы $A$. Мы налагаем ограничение (17) на входные состояния канала $\Phi^{\otimes n}$, что эквивалентно аналогичному ограничению для канала $\Phi^{\otimes n} \otimes \operatorname{Id}_{B}^{\otimes n}$ с операторами $F_{A B}^{(n)}=F^{(n)} \otimes I_{B}^{\otimes n}$. Обозначим $\mathscr{P}_{A B}^{(n)}$ совокупность пар $\left(\pi^{(n)}, \Sigma^{(n)}\right)$, где $\pi^{(n)}=\left\{\pi_{w}^{(n)}\right\}-$ распределение вероятностей, а $\Sigma^{(n)}=\left\{S_{w}^{(n)}\right\}$ - множество состояний вида $(29)$, удовлетворяющих ограничению (19) с операторами $F_{A B}^{(n)}$. Классическая пропускная способность такого протокола и называется пропускной способностью с использованием сцепленного состояния $C_{\text {еа }}(\Phi)$ канала $\Phi$ при ограничении (17).

Пусть $S$ - оператор плотности, для которого энтропии $H(S)$ и $H(\Phi(S))$ конечны, тогда квантовая взаимная информаиия определяется формулой

$$
I(S, \Phi)=H(S)+H(\Phi(S))-H(S ; \Phi)
$$

где $H(S ; \Phi)=H\left(\left(\Phi \otimes \operatorname{Id}_{R}\right)[|\psi\rangle\langle\psi|]\right)$ - обменная энтропия, $|\psi\rangle\langle\psi|$ - очищение состояния $S$, а $R$ - очищающая система (см., например, [5]). Можно также представить взаимную информацию как относительную энтропию

$$
I(S, \Phi)=H\left(\left(\Phi \otimes \operatorname{Id}_{R}\right)[|\psi\rangle\langle\psi|] ; \Phi[S] \otimes S\right) .
$$

Это выражение определено, даже если слагаемые в (30) бесконечны.

Если оператор ограничения $F$ удовлетворяет условию (9), то энтропия $H(S)$ конечна для всех $S$, удовлетворяющих ограничению $\operatorname{Tr} S F \leqslant E$. В самом деле,

$$
\beta \operatorname{Tr} S F-H(S)=H\left(S ; S_{\beta}\right)-\ln \operatorname{Tr} \exp (-\beta F),
$$

откуда

$$
H(S) \leqslant \beta E+\ln \operatorname{Tr} \exp (-\beta F) .
$$

Предложение 4. Пусть $\Phi$ - канал, удовлетворяющий условию (16) с оператором $F$, удовлетворяющим условию (9). Тогда его классическая пропускная способность с использованием счепленного состояния при ограничении (17) конечна и равна

$$
C_{e a}(\Phi)=\sup _{S: \operatorname{Tr} S F \leqslant E} I(S, \Phi)
$$


Д о к а з а т е л ь с т в о. Модифицируя доказательство предложения 2 , получаем

$$
C_{e a}(\Phi)=\lim _{n \rightarrow \infty} \frac{1}{n} C_{e a}^{(n)}(\Phi)
$$

где

$$
C_{e a}^{(n)}(\Phi)=\sup _{\left(\pi^{(n)}, \Sigma^{(n)}\right) \in \mathscr{P}_{A B}^{(n)}} \chi\left(\left\{\pi_{w}^{(n)}\right\},\left\{\left(\Phi^{\otimes n} \otimes \operatorname{Id}_{B}^{\otimes n}\right)\left[S_{w}^{(n)}\right]\right\}\right) .
$$

Заметим, что все члены в выражении $\chi$ конечны. В самом деле, в силу субаддитивности квантовой энтропии, получаем, как в работе [3]:

$$
H\left(\sum_{w=1}^{N} \pi_{w}^{(n)}\left(\Phi^{\otimes n} \otimes \operatorname{Id}_{B}^{\otimes n}\right)\left[S_{w}^{(n)}\right]\right) \leqslant H\left(\Phi^{\otimes n}\left[\sum_{w=1}^{N} \pi_{w}^{(n)} S_{w A}^{(n)}\right]\right)+H\left(S_{B}^{(n)}\right),
$$

где $S_{w A}^{(n)}$ - частичное состояние $S_{w}^{(n)}$ в подсистеме $A$, что является конечным в силу предполагаемой конечности энтропии $H\left(S_{B}^{(n)}\right)$ и условия $(16)$.

Докажем сначала неравенство $\leqslant$ в (34). Как показано в [3], выражение $\chi$ в правой части (36) ограничено сверху величиной $I\left(\sum_{w=1}^{N} \pi_{w}^{(n)} S_{w A}^{(n)}, \Phi^{\otimes n}\right)$. Из (35) мы получаем

$$
C_{e a}(\Phi) \leqslant \lim _{n \rightarrow \infty} \frac{1}{n} \sup _{\left(\pi^{(n)}, \Sigma^{(n)}\right) \in \mathscr{P}_{A B}^{(n)}} I\left(\sum_{w=1}^{N} \pi_{w}^{(n)} S_{w A}^{(n)}, \Phi^{\otimes n}\right) .
$$

Правая часть не превосходит величины

$$
\sup _{S^{(n)}: \operatorname{Tr} S^{(n)} F^{(n)} \leqslant n E} I\left(S^{(n)}, \Phi^{\otimes n}\right) \equiv \bar{I}_{n}(\Phi) .
$$

Однако последовательность $\bar{I}_{n}(\Phi)$ аддитивна; достаточно доказать лишь, что

$$
\bar{I}_{n}(\Phi) \leqslant n \bar{I}_{1}(\Phi) .
$$

В самом деле, в силу субаддитивности квантовой взаимной информации,

$$
I\left(S^{(n)}, \Phi^{\otimes n}\right) \leqslant \sum_{j=1}^{n} I\left(S_{j}^{(n)}, \Phi\right)
$$

где $S_{j}^{(n)}$ - частичные состояния, и, в силу вогнутости,

$$
\sum_{j=1}^{n} I\left(S_{j}^{(n)}, \Phi\right) \leqslant n \sum_{j=1}^{n} I\left(\frac{1}{n} \sum_{j=1}^{n} S_{j}^{(n)}, \Phi\right) .
$$

Однако неравенство $\operatorname{Tr} S^{(n)} F^{(n)} \leqslant n E$ эквивалентно $\operatorname{Tr}\left(n^{-1} \sum_{j=1}^{n} S_{j}^{(n)}\right) F \leqslant$ $E$, откуда вытекает (37). Таким образом,

$$
C_{e a}(\Phi) \leqslant \sup _{S: \operatorname{Tr} S F \leqslant E} I(S, \Phi)
$$


Теперь докажем обратное неравенство. Поскольку $F$ не пропорционален единичному оператору, образ выпуклого множества всех операторов плотности при отображении $S \rightarrow \operatorname{Tr} S F$ содержит интервал. Предположим сначала, что $E$ не является минимальным собственным значением оператора $F$. Тогда найдутся вещественное число $E^{\prime}$ и оператор плотности $S$ в $\mathscr{H}_{A}$ такие, что $\operatorname{Tr} S F \leqslant E^{\prime}<E$. Рассмотрим спектральное разложение $S=\sum_{j=1}^{\infty} \lambda_{j}\left|e_{j}\right\rangle\left\langle e_{j}\right|$ и обозначим $S_{d}=\sum_{j=1}^{d} \tilde{\lambda}_{j}\left|e_{j}\right\rangle\left\langle e_{j}\right|$, где $\tilde{\lambda}_{j}=\left(\sum_{k=1}^{d} \lambda_{k}\right)^{-1} \lambda_{j}-$ собственные значения оператора плотности $S_{d}$. Тогда $\left\|S-S_{d}\right\|_{1} \rightarrow 0$ при $d \rightarrow \infty$, где $\|\cdot\|_{1}-$ следовая норма. Положим $f(j)=\left\|\sqrt{F} e_{j}\right\|^{2}$, тогда

$$
\operatorname{Tr} S_{d} F=\sum_{j=1}^{d} \tilde{\lambda}_{j} f(j)=E^{\prime}+\varepsilon_{d}
$$

где $\varepsilon_{d} \rightarrow 0$ при $d \rightarrow \infty$.

Рассмотрим теперь сильно типичный проектор $P^{n, \delta}$ оператора плотности $S_{d}^{\otimes n}$, который определяется следующим образом. Собственные значения и собственные векторы оператора $S_{d}^{\otimes n}$ суть $\tilde{\lambda}_{J}=\tilde{\lambda}_{j_{1}} \cdots \tilde{\lambda}_{j_{n}}$, $\left|e_{J}\right\rangle=\left|e_{j_{1}}\right\rangle \otimes \cdots \otimes\left|e_{j_{n}}\right\rangle$, где $J=\left(j_{1}, \ldots, j_{n}\right)$. Фиксируем малое положительное $\delta$. Последовательность $J$ называется сильно типичной [10], если числа $N(j \mid J)$ появления символов $j$ в $J$ удовлетворяют условию

$$
\left|\frac{N(j \mid J)}{n}-\tilde{\lambda}_{j}\right|<\delta, \quad j=1, \ldots, d,
$$

и $N(j \mid J)=0$, если $\tilde{\lambda}_{j}=0$. Обозначим совокупность всех сильно типичных последовательностей через $B^{n, \delta}$, и пусть $\mathbf{P}^{n}-$ распределение вероятностей, задаваемое собственными значениями $\tilde{\lambda}_{J}$. Согласно закону больших чисел, $\mathbf{P}^{n}\left(B^{n, \delta}\right) \rightarrow 1$ при $n \rightarrow \infty$. Для произвольной функции $g(j), j=1, \ldots, d$, и последовательности $J=\left(j_{1}, \ldots, j_{n}\right) \in B^{n, \delta}$ выполняется

$$
\left|\frac{g\left(j_{1}\right)+\cdots+g\left(j_{n}\right)}{n}-\sum_{j=1}^{d} \tilde{\lambda}_{j} g(j)\right|<\delta \max \{g(j) ; j=1, \ldots, d\} .
$$

Сильно типичный проектор оператора плотности $S_{d}^{\otimes n}$ определяется как $P^{n, \delta}=\sum_{J \in B^{n, \delta}}\left|e_{J}\right\rangle\left\langle e_{J}\right|$. Обозначим $d_{n, \delta}=\operatorname{dim} P^{n, \delta}, \bar{S}_{d}^{n, \delta}=P^{n, \delta} / d_{n, \delta}$. Благодаря сильной типичности,

$$
\begin{aligned}
& \left|\operatorname{Tr}\left(\bar{S}_{d}^{n, \delta}-S_{d}^{\otimes n}\right) F^{(n)}\right| \\
& \quad=\left|n \operatorname{Tr} \frac{(F \otimes I \otimes \cdots \otimes I+\cdots+I \otimes \cdots \otimes I \otimes F)}{n}\left(\bar{S}^{n, \delta}-S_{d}^{\otimes n}\right)\right| \\
& \quad=\frac{n}{d_{n, \delta}}\left|\sum_{J \in B^{n, \delta}}\left[\frac{f\left(j_{1}\right)+\cdots+f\left(j_{n}\right)}{n}-\sum_{j=1}^{d} \tilde{\lambda}_{j} f(j)\right]\right| \\
& \quad \leqslant n \delta \max \{f(j) ; j=1, \ldots, d\},
\end{aligned}
$$


откуда

$$
\begin{aligned}
\operatorname{Tr} \bar{S}_{d}^{n, \delta} F^{(n)} & \leqslant \operatorname{Tr} S_{d}^{\otimes n} F^{(n)}+n \delta \max \{f(j) ; j=1, \ldots, d\} \\
& =n\left(E^{\prime}+\varepsilon_{d}+\delta \max \{f(j) ; j=1, \ldots, d\}\right)
\end{aligned}
$$

Для любого достаточно большого $d$ можно найти $\delta_{0}$ такое, что правая часть будет меньше или равна $n E$ при $\delta \leqslant \delta_{0}$. Тогда, используя выражение (36) и конкретный протокол кодирования, описываемый ниже, мы сможем доказать, что

$$
C_{e a}^{(n)}(\Phi) \geqslant I\left(\bar{S}_{d}^{n, \delta}, \Phi^{\otimes n}\right)
$$

В самом деле, пусть

$$
\left|\psi_{A B}\right\rangle=\frac{1}{\sqrt{d_{n, \delta}}} \sum_{J \in B^{n, \delta}}\left|e_{J}\right\rangle \otimes\left|e_{J}\right\rangle
$$

Как показано в [2], [3], существует система унитарных операторов $W_{\alpha \beta}$; $\alpha, \beta=1, \ldots, d_{n, \delta}$, в $\mathscr{H}_{A}$ такая, что $\left(W_{\alpha \beta} \otimes I_{B}\right)\left|\psi_{A B}\right\rangle$ является ортонормированной системой в $\mathscr{H}_{A} \otimes \mathscr{H}_{B} ;$ более того,

$$
\sum_{\alpha, \beta=1}^{d_{n, \delta}}\left(W_{\alpha \beta} \otimes I_{B}\right)\left|\psi_{A B}\right\rangle\left\langle\psi_{A B}\right|\left(W_{\alpha \beta} \otimes I_{B}\right)^{*}=P^{n, \delta} \otimes P^{n, \delta}
$$

Пусть передается классический сигнал $w=(\alpha, \beta)$ с равными вероятностями $\pi_{w}=1 / d_{n, \delta}^{2}$, сцепленное состояние выбрано как $S_{A B}=\left|\psi_{A B}\right\rangle\left\langle\psi_{A B}\right|$ и кодирование - как $\mathscr{E}_{A}^{w}[S]=W_{\alpha \beta} S W_{\alpha \beta}^{*}$. Такой выбор удовлетворяет ограничению на входе, поскольку (41) влечет

$$
\sum_{w} \pi_{w} \mathscr{E}_{A}^{w}\left[S_{A B}\right]=\bar{S}_{d}^{n, \delta} \otimes \bar{S}_{d}^{n, \delta}
$$

Таким образом, для этого конкретного протокола условие $\left(\pi^{(n)}, \Sigma^{(n)}\right) \epsilon$ $\mathscr{P}_{A B}^{(n)}$ в (36) эквивалентно тому, что $\operatorname{Tr} \bar{S}_{d}^{n, \delta} F^{(n)} \leqslant n E$. Тогда мы получаем

$$
\begin{aligned}
C_{e a}^{(n)}(\Phi) \geqslant & H\left(\frac{1}{\left(d^{n, \delta}\right)^{2}} \sum_{\alpha, \beta}\left(\Phi^{\otimes n} \otimes \operatorname{Id}_{B}^{\otimes n}\right)\left[S_{A B}^{\alpha \beta}\right]\right) \\
& -\frac{1}{\left(d^{n, \delta}\right)^{2}} \sum_{\alpha, \beta} H\left(\left(\Phi^{\otimes n} \otimes \operatorname{Id}_{B}^{\otimes n}\right)\left[S_{A B}^{\alpha \beta}\right]\right)
\end{aligned}
$$

где $S_{A B}^{\alpha \beta}=\left(W_{\alpha \beta} \otimes I_{B}\right)\left|\psi_{A B}\right\rangle\left\langle\psi_{A B}\right|\left(W_{\alpha \beta} \otimes I_{B}\right)^{*}$. Согласно (42), первое слагаемое в правой части равно $H\left(\left(\Phi \otimes \operatorname{Id}_{B}\right)\left[\bar{S}_{d}^{n, \delta} \otimes \bar{S}_{d}^{n, \delta}\right]\right)=H\left(\bar{S}_{d}^{n, \delta}\right)+$ $H\left(\Phi\left[\bar{S}_{d}^{n, \delta}\right]\right)$. Поскольку $S_{A B}^{\alpha \beta}$ является очишением состояния $\bar{S}_{d}^{n, \delta}$ в $\mathscr{H}_{B}$, 
энтропии во втором слагаемом равны $H\left(\bar{S}_{d}^{n, \delta}, \Phi\right)$. В силу выражения для квантовой взаимной информации (30), отсюда следует (40).

Переходя к пределу $n \rightarrow \infty, \delta \rightarrow 0$ и используя предельное соотношение из [3], мы получаем

$$
C_{e a}(\Phi)=\lim _{n \rightarrow \infty} \frac{1}{n} C_{e a}^{(n)}(\Phi) \geqslant I\left(S_{d}, \Phi\right),
$$

где $\operatorname{Tr} S_{d} F=E^{\prime}+\varepsilon_{d} \leqslant E$. Наконец, переходя к пределу $d \rightarrow \infty$, мы докажем, что

$$
\liminf _{d \rightarrow \infty} I\left(S_{d}, \Phi\right) \geqslant I(S, \Phi) .
$$

Чтобы это показать, представим взаимную информацию как квантовую относительную энтропию (31), где $|\psi\rangle\langle\psi|$ - очищение состояния $S, R$ - очищающая система, и аналогично для $I\left(S_{d}, \Phi\right)$. Если $|\psi\rangle=\sum_{j=1}^{\infty} \sqrt{\lambda_{j}}\left|e_{j}\right\rangle \otimes\left|e_{j}\right\rangle$, то

$$
\left|\psi_{d}\right\rangle=\sum_{j=1}^{d} \sqrt{\tilde{\lambda}_{j}}\left|e_{j}\right\rangle \otimes\left|e_{j}\right\rangle
$$

является очищением состояния $S_{d}$. Имеем $\||\psi\rangle-\left|\psi_{d}\right\rangle \| \rightarrow 0$, и поэтому

$$
\||\psi\rangle\left\langle\psi|-| \psi_{d}\right\rangle\left\langle\psi_{d}\right| \|_{1} \rightarrow 0 \quad \text { при } \quad d \rightarrow \infty
$$

следовательно, (43) вытекает из полунепрерывности снизу относительной энтропии [8]. Таким образом, мы получаем

$$
C_{e a}(\Phi) \geqslant I(S, \Phi)
$$

где $S$ - произвольный оператор плотности, удовлетворяющий условию $\operatorname{Tr} S F<E$. Это легко распространяется на операторы с $\operatorname{Tr} S F=E$ путем аппроксимации их операторами $S_{\epsilon}=(1-\epsilon) S+\epsilon|e\rangle\langle e|$, где вектор $e$ выбран так, что $\langle e|F| e\rangle<E$.

В случае, когда $E$ - минимальное собственное значение оператора $F$, условие $\operatorname{Tr} S F \leqslant E$ сводится к тому, что носитель $S$ содержится в спектральном проекторе оператора $F$, отвечающем этому минимальному значению. Из условия (9) вытекает, что спектр оператора $F$ состоит из собственных значений конечной кратности. Поэтому носитель $S$ конечномерен, и мы можем считать $S_{d}=S$. Тогда мы можем повторить рассуждения с сохранением равенства $\operatorname{Tr} S F=E$ на каждом шаге. Итак, мы установили соотношение

$$
C_{e a}(\Phi) \geqslant \sup _{S: \operatorname{Tr} S F \leqslant E} I(S, \Phi)
$$

и, значит, доказали равенство в (34). Предложение 4 доказано. 
Теперь мы исследуем вопрос - когда достигается верхняя грань в правой части соотношения (34). Заметим, что условия следующей леммы выполняются для любого оператора, удовлетворяющего условию (9).

Лемма. Пусть спектр оператора $F$ состоит из собственных значений $f_{n}$ конечной кратности $u \lim _{n \rightarrow \infty} f_{n}=+\infty$, тогда множество операторов плотности $\mathfrak{S}_{E}:=\{S: \operatorname{Tr} S F \leqslant E\}$ компактно.

Д ок аз а т е з с т в о. Напомним, что, согласно [7], слабая сходимость в множестве операторов плотности равносильна сходимости по следовой норме. Множество $\mathfrak{S}_{E}$ замкнуто, поскольку для любой последовательности $\left\{S_{n}\right\} \subset \mathfrak{S}_{E}$, сходящейся к оператору плотности $S$, имеем $\lim _{n \rightarrow \infty} \operatorname{Tr} S_{n} F \geqslant \operatorname{Tr} S F$.

Не ограничивая общности, можно предположить, что последовательность $f_{n}$ монотонно возрастает. Обозначим $P_{n}$ конечномерный проектор на собственное подпространство, отвечающее первым $n$ собственным значениям, тогда $P_{n} \uparrow I$. Согласно общему критерию (некоммутативный аналог критерия из [9]), подмножество $\mathfrak{S}^{\prime}$ операторов плотности относительно слабо компактно тогда и только тогда, когда для любого $\varepsilon>0$ найдется конечномерный проектор $P$ такой, что $\operatorname{Tr} S(I-P) \leqslant \varepsilon$ для всех $S \in \mathfrak{S}^{\prime}$, см. п. III.9 в [12].

Поскольку $f_{n+1}\left(I-P_{n}\right) \leqslant F$, имеем $\operatorname{Tr} S\left(I-P_{n}\right) \leqslant f_{n+1}^{-1} \operatorname{Tr} S F \leqslant$ $f_{n+1}^{-1} E$ при $S \in \mathfrak{S}_{E}$, откуда и следует утверждение леммы.

Рассмотрим теперь условия, при которых взаимная информация полунепрерывна сверху и, значит, достигает точной верхней грани на компакте $\mathfrak{S}_{E}$.

Предложение 5. Пусть оператор $F$ удовлетворяет условию (9) и найдется самосопряженный оператор $\widetilde{F}$, удовлетворяющий (9) и такой, что $\Phi^{*}[\widetilde{F}] \leqslant F$, где $\Phi^{*}-$ сопряженный канал. Тогда

$$
C_{e a}(\Phi)=\max _{S: \operatorname{Tr} S F \leqslant E} I(S, \Phi) .
$$

Д о к а з а т е л ь с в о. Займемся отдельно каждым слагаемым в формуле (30). Напомним, что квантовая энтропия полунепрерывна снизу. Поскольку обменная энтропия может быть представлена в виде $H(S ; \Phi)=H\left(\Phi_{E}[S]\right)$, где $\Phi_{E}-$ канал из пространства системы $\mathscr{H}_{A}$ в пространство окружения $\mathscr{H}_{E}$ [2], [5], она также полунепрерывна снизу, и поэтому последний член в выражении (30) полунепрерывен сверху. Относительно первого слагаемого известно [8], что оно полунепрерывно сверху (и, следовательно, непрерывно) на множестве $\mathfrak{S}_{E}=$ $\{S: \operatorname{Tr} S F \leqslant E\}$, если оператор $F$ удовлетворяет условию (9). Доказательство полунепрерывности сверху проходит следующим образом: имеем

$$
\beta \operatorname{Tr} S_{n} F-H\left(S_{n}\right)=H\left(S_{n} ; S_{\beta}\right)-\ln \operatorname{Tr} \exp (-\beta F)
$$


и аналогично для $S$ вместо $S_{n}$. Используя полунепрерывность снизу относительной энтропии, получаем

$$
H(S) \geqslant \limsup _{n \rightarrow \infty} H\left(S_{n}\right)-\beta \limsup _{n \rightarrow \infty} \operatorname{Tr} S_{n} F .
$$

Для $S_{n} \in \mathfrak{S}_{E}$ последний член $\geqslant-\beta E$, что может быть сделано сколь угодно малым.

Мы можем применить аналогичное рассуждение ко второму слагаемому в (30) в предположении, что существует самосопряженный оператор $\widetilde{F}$, удовлетворяющий условию $(9)$ и такой, что $\Phi^{*}[\widetilde{F}] \leqslant F$; соотношение (45) тогда заменяется на

$$
\beta \operatorname{Tr} S_{n} F-H\left(\Phi\left[S_{n}\right]\right) \geqslant H\left(\Phi\left[S_{n}\right] ; \widetilde{S}_{\beta}\right)-\ln \operatorname{Tr} \exp (-\beta \widetilde{F}),
$$

где $\widetilde{S}_{\beta}=[\operatorname{Tr} \exp (-\beta \widetilde{F})]^{-1} \exp (-\beta \widetilde{F})$, и доказательство проходит аналогичным образом. Более того, это предположение влечет также выполнение условия (16). В самом деле, обозначая $\Phi[S]=S^{\prime}$ и используя (33), имеем

$$
\sup _{S: \operatorname{Tr} S F \leqslant E} H(\Phi[S]) \leqslant \sup _{S^{\prime}: \operatorname{Tr} S^{\prime} \widetilde{F} \leqslant E} H\left(S^{\prime}\right) \leqslant \theta E+\ln \operatorname{Tr} \exp (-\theta \tilde{F}) .
$$

Предложение 5 доказано.

Такой набор условий, гарантирующий достижимость супремума в (34), выполняется, например, в случае, когда $\Phi-$ бозонный гауссовский канал, а $F$ - положительный квадратичный многочлен от канонических наблюдаемых, например, оператор энергии. Выигрыш от использования сцепленности $G=C_{e a}(\Phi) / \bar{C}^{(1)}(\Phi)$ был подсчитан в [5]. В частности, для канала (28), когда среднее число фотонов сигнала $E$ стремится к нулю, а $N>0$,

$$
\bar{C}^{(1)}(\Phi) \sim E \ln \left(\frac{N+1}{N}\right), \quad C_{e a}(\Phi) \sim-E \ln E /(N+1),
$$

и $G$ стремится к бесконечности, как $-\ln E$.

В этой работе нас интересовал случай, когда все энтропии, входящие в выражение для пропускной способности, конечны, что обеспечивалось условиями (16), (9). Путем надлежащей аппроксимации можно получить в общем случае выражение для $C_{e a}(\Phi)$, даваемое формулой $(34)$, где $I(S, \Phi)$ определяется, как в $(31)$.

Приложение. Оператором плотности $S$ в гильбертовом пространстве $\mathscr{H}$ называется положительный оператор с единичным следом. Будучи компактным оператором, $S$ допускает спектральное разложение вида

$$
S=\sum_{j=1}^{\infty} \lambda_{j}\left|e_{j}\right\rangle\left\langle e_{j}\right|
$$


где $\lambda_{j}$ - собственные значения, образующие распределение вероятностей, а $e_{j}$ - ортонормированные собственные векторы. Энтропия $S$ определяется выражением $H(S)=-\sum_{j=1}^{\infty} \lambda_{j} \ln \lambda_{j}$ и равна $-\operatorname{Tr} S \ln S$, если след конечен.

Для двух операторов плотности $S, S^{\prime}$ квантовая относительная энтропия определяется соотношением

$$
H\left(S ; S^{\prime}\right)=\sum_{j, k=1}^{\infty}\left|\left\langle e_{k}^{\prime} \mid e_{j}\right\rangle\right|^{2} \lambda_{j}\left(\ln \lambda_{j}-\ln \lambda_{k}^{\prime}\right)
$$

где $\lambda_{k}^{\prime}, e_{k}^{\prime}$ - собственные значения и собственные векторы оператора $S^{\prime}$. Это выражение определено корректно и принимает значения в $[0, \infty]$ в силу неравенства $\lambda(\ln \lambda-\ln \mu) \geqslant \lambda-\mu$ при $\lambda, \mu \geqslant 0$. В случае конечности следа $H\left(S ; S^{\prime}\right)=\operatorname{Tr} S\left(\ln S-\ln S^{\prime}\right)$. Как энтропия, так и относительная энтропия - полунепрерывные снизу функции от $S, S^{\prime}$ относительно следовой нормы $\|T\|_{1}=\operatorname{Tr}|T|[8]$.

\section{СПИСОК ЛИТЕРАТУРЫ}

1. Bennett C. H., Shor P. W., Smolin J. A., Thapliyal A. V. Entanglement-assisted classical capacity of noisy quantum channel. - Phys. Rev. Lett., 1999, v. 83, p. 30813084; LANL Report quant-ph/9904023.

2. Bennett C. H., Shor P. W., Smolin J. A., Thapliyal A. V. Entanglement-assisted capacity and the reverse Shannon theorem. - LANL Report quant-ph/0106052.

3. Holevo A. S. On entanglement-assisted classical capacity. - J. Math. Phys., 2002, v. 43, p. 4326-4333; LANL Report quant-ph/0106075.

4. Холево A. C. Введение в квантовую теорию информации. М.: МЦНМО, 2002, $126 \mathrm{c}$.

5. Holevo A. S., Werner R. F. Evaluating capacities of Bosonic Gaussian channels. Phys. Rev. A, 2000, v. 63, art. 032313; LANL Report quant-ph/9912067.

6. Холево A. C. Квантовые теоремы кодирования. - Успехи матем. наук, 1998, т. 53, № 6, c. 193-230; LANL Report quant-ph/9808023.

7. Dell'Antonio G. F. On the limits of sequences of normal states. - Comm. Pure Appl. Math., 1967, v. 20, p. 413-430.

8. Wehrl A. General properties of entropy. - Rev. Modern. Phys., 1978, v. 50, p. 221260.

9. Прохоров Ю. В. Случайные процессы и предельные теоремы теории вероятностей. - Теория вероятн. и ее примен., 1956, т. 1, в. 2, с. 177-235.

10. Чисар И., Кёрнер Я. Теория информации. Теоремы кодирования для дискретных систем без памяти. М.: Мир, 1985, 397 с.

11. Matsumoto K., Shimono T., Winter A. Remarks on additivity of the Holevo capacity and of the entanglement of formation. - LANL Report quant-ph/0206148.

12. Сарьмсаков T. А. Введение в квантовую теорию вероятностей. Ташкент: Фан, $1985,184 \mathrm{c}$.

13. Hayashi M., Nagaoka $H$. General formulas for capacity of classical-quantum channels. - LANL Report quant-ph/0206186. 